\title{
Modulation of Sodium-Dependent Transporters Expression in Normal Human Keratinocytes by a Sodium Rich Isotonic Thermal Water
}

\author{
Clotilde Verdy $^{1}$, Jean-Eric Branka ${ }^{1 *}$, Luc Lefeuvre ${ }^{2}$ \\ ${ }^{1}$ Effiscience, Immeuble Bio Ouest, Nantes, France; ${ }^{2}$ Laboratoires Dermatologiques d'Uriage, Siège Social, Courbevoie, France. \\ Email: ${ }^{*}$ jebranka@effiscience.fr
}

Received July $25^{\text {th }}, 2012$; revised August $26^{\text {th }}, 2012$; accepted September $6^{\text {th }}, 2012$

\begin{abstract}
Background/Aim: In order to show that water can participate to the skin defense in front of different stress, we investigated the effect of an isotonic thermal water notably rich in Sodium (i.e. the Uriage thermal water) on 1) The taurine transporter (TauT) expression in human normal keratinocytes irradiated or not by UVB; and 2) the Sodium-dependent vitamin C transporter 1 (SVCT1) expression in human normal keratinocytes issued from two "young" and two "aged" subjects, irradiated or not by UVB. Methods and Results: Using sensible and specific TAUT and SVCT1 ELISA assays developed in house, we provide 1) the unambiguous demonstration that the Uriage thermal water is able to help the epidermis to maintain its taurine content under UVB irradiation; 2) the first example of an altered SVCT1 expression in "aged" keratinocytes and of a significant positive effect of the Uriage thermal water on this altered SVCT1 production; and 3) arguments showing that Uriage thermal water is also able to participate to the regulation of the SVCT1 production in UVB-irradiated keratinocytes. Conclusion: Taking together, these results suggest that the Uriage thermal water could act to efficiently protect the skin from dehydration through its effect on TauT and SVCT1 expression, and furthermore, to allow a more efficient taurine and ascorbic acid supplying to the epidermis in order to protect him from other aggressions such as oxidant stress for example.
\end{abstract}

Keywords: TauT; SVCT1; Thermal Water; Young and Aged Keratinocytes; UVB

\section{Introduction}

Skin is daily exposed to external aggressions such as UV irradiations, pollutants, etc. In order to ensure its protection in front of these stress, the skin has developed several defense mechanisms including natural physical UV filters, i.e. the urocanic acid [1], physiological systems of protection involving the melanin pigmentation [2] and the thickening of the epidermal horny layer [3] and enzymatic and non enzymatic systems [4] able to fight against oxidative stress induced by UV and/or pollutants notably. Less visible than UV or pollutants but also very important, particular environmental conditions can also seriously damage the cutaneous tissue. Thus, a particularly dry environment for example, can greatly dehydrates the skin by notably down regulating the expression of the aquaporin-3 [5], a protein forming pores and selectively conducting water molecules through the cellular membrane. In such cases of osmotic perturbations, the cutaneous cells ensure their own protection through the

${ }^{*}$ Corresponding author. accumulation of organic osmolytes such as taurine, a molecule implicated in the cell volume regulation and in the defense from dehydration [6].

Taurine, and other molecules implicated in these defense mechanisms against oxidative and dehydrating stress, however need specialized transporters to penetrate into the cell. It is the case for example of the ascorbic acid, a well known anti-oxidant compound also named vitamin $\mathrm{C}$. Thus, the vitamin $\mathrm{C}$ will be introduced into the cell by the Sodium-dependent Vitamine C Transporters (SVCT) types 1 and 2 (and more specifically by the type 1 in the epidermis) [7], whereas the taurine will penetrate the cell through Taurine Transporters (TauT), whose one type is also Sodium-dependant (an another type of TauT, a Chloride-dependent one, is also expressed in the keratinocytes) [8]. As we can see, organic osmolytes and mineral elements seem to be of a great importance in the defense of the skin in front of different physico-chemical stress, not only by holding the water inside the cell as the taurine do for example, but also by regulating mechanisms providing some essentials "shield 
compounds" to the cell (i.e. the ascorbic acid and the taurine), as the Sodium does.

In addition, inorganic osmolytes can regulate, not only the functioning of membrane transporters, but also their expression. Indeed, Zinc and Cobalt ions have been demonstrated to be able to increase the SVCT-2 expression in osteoblasts [9] and in brain cortical capillary endothelial cells [10] respectively, whereas Manganese has been shown to increase TauT mRNA levels in rat astrocytes [11].

Reasoning that hydrating cosmetic products not only contain oily compounds but also water which itself contains ions like Sodium, Zinc, etc., we examined if an isotonic water particularly rich in Sodium, calcium, magnesium, manganese, zinc, copper and silicium (i.e. the Uriage thermal water), could provide efficient protection to the skin in front of dehydration notably, by acting on the keratinocyte expression of TauT.

As previously published works mainly consisted in immunohistochemical and/or mRNA analysis approaches, and in order to provide unambiguous quantitative results, we have chosen to analyze the effect of the Uriage thermal water on the TauT expression at the protein level by developing specific and sensible ELISA assay. TauT expression was then studied in normal human keratinocytes at a basal level but also in stressful conditions, by irradiating the cells with UVB.

In the second part of this work, we examined the effect of the Uriage thermal water on the expression of the SVCT-1 in human normal keratinocytes, taking in account that ascorbic acid has been implicated in the skin defense against oxidative stress [12], in the regulation of the production of molecules implicated in the skin hydration [13], and in the diminution of the transepidermal water loss [14], we also examined the effect of the Uriage thermal water on the expression of the SVCT-1 in human normal keratinocytes. Following the same experimental design than those followed in the TauT expression study, we developed a specific and sensible ELISA assay to quantify SVCT-1 in human normal keratinocytes at a basal level and after UVB irradiation. In addition and in a very interesting way, we have chosen to consider intrinsic aging as a particular type of stress, and we also studied the effect of the Uriage thermal water on the SVCT-1 expression in keratinocytes issued from young and old subjects.

\section{Materials and Methods}

\subsection{Reagents and Materials}

Human normal keratinocytes were issued from four donors aged of 32, 35, 66 and 74 years. Keratinocytes growth medium (KGM) was purchased from Promocell
(Heidelberg, Germany). Phorbol myristate acetate, phosphate buffered solution and $\mathrm{FeCl}_{3}$ came from Sigma-Aldrich (St. Louis, USA). Rat TAU1 control peptide, rabbit anti-rat TAU1 antiserum, human SVCT control peptide and rabbit anti-human SVCT IgG were purchased from Alpha Diagnostic International (San Antonio, USA). BSA fraction V and Tween 20 came from Acros Organics (Geel, Belgium). Goat anti-rabbit IgG (H\&L) horseradish peroxidase conjugated was purchased from Antibodies-online (Aachen, Germany). Substrate reagent for the peroxidase came from R\&D Systems (Minneapolis, USA). $\mathrm{H}_{2} \mathrm{SO}_{4}$ came from Merck (Darmstadt, Germany). Multiwell culture plates and EIA/RIA Stripwell plates were purchased from Corning Costar (Brumath, France). Thermal water came from Uriage-les-Bains, France.

Uriage thermal water: Ionic composition and osmolarity

Mean ionic content per liter:

$\begin{array}{lll}\text { Sulfates } & 2860 \mathrm{mg} \text { Potassium } & 45.5 \mathrm{mg} \\ \text { Chlorures } & 3500 \mathrm{mg} \text { Silicon } & 42 \mathrm{mg} \\ \text { Sodium } & 2360 \mathrm{mg} \text { Zinc } & 160 \mu \mathrm{g} \\ \text { Bicarbonate } & 390 \mathrm{mg} \text { Manganese } & 154 \mu \mathrm{g} \\ \text { Calcium } & 600 \mathrm{mg} \text { Copper } & 75 \mu \mathrm{g} \\ \text { Magnesium } & 125 \mathrm{mg} \text { Iron } & 15 \mu \mathrm{g} \\ \text { Osmolarity of } & \text { Uriage thermal water is } 275 \pm 40 \\ \text { Osm. }\end{array}$

\subsection{Cell Cultures and Irradiation}

Human Normal human keratinocytes were cultured in Keratinocyte Growth Medium (KGM) in a humidified incubator under a $5 \% \mathrm{CO}_{2}, 95 \%$ air atmosphere.

For the study of TauT expression, confluent monolayers of keratinocytes were incubated in the absence (control) or in the presence of increasing concentrations $(0.1$; 1 and $10 \%, \mathrm{v} / \mathrm{v}$ ) of the Uriage thermal water for 72 hours. At the indicated time-point (24 and $72 \mathrm{~h}$ ), monolayers were rinsed with a Phosphate Buffered Solution (PBS) and stored at $-20^{\circ} \mathrm{C}$ until the TauT quantification.

For the study of SVCT-1 expression, confluent monolayers of keratinocytes were incubated in the absence (control) or in the presence of increasing concentrations $(0.1 ; 1$ and $10 \%, \mathrm{v} / \mathrm{v})$ of the Uriage thermal water for a 24 hours period. At the end of the incubation period, monolayers were rinsed with $\mathrm{PBS}$ and stored at $-20^{\circ} \mathrm{C}$ until the SVCT-1 quantification.

In case of UV exposure, the cells were irradiated with $30 \mathrm{~mJ} / \mathrm{cm}^{2}$ UVB with a UV lamp (Vuibert Lourmat).

\subsection{TAUT and SVCT-1 ELISA Assay}

Multiwell plates especially designed to perform ELISA assays were incubated overnight at $4^{\circ} \mathrm{C}$ with $100 \mu \mathrm{l}$ of serial dilutions of the standard peptide or of the samples 
to assay. At the end of this incubation period, non-specific binding sites were saturated by the addition of 300 $\mu l$ per well of a $1 \%$ BSA solution in PBS. After an incubation period of one hour at room temperature, wells were emptied and filled with $100 \mu \mathrm{l}$ of a $1 \%$ BSA solution in PBS containing $0.1 \%(\mathrm{v} / \mathrm{v})$ of the TauT or the SVCT-1 antibody. After an incubation period of two hours at room temperature, the wells were washed three times with PBS containing $0.1 \%$ of Tween 20 . Wells were then filled with $100 \mu \mathrm{l}$ of a $1 \%$ BSA solution in PBS containing the secondary antibody (dilution: 1/1000) coupled to a peroxidase and were incubated at room temperature for a two hours period. At the end of this incubation period, wells were again washed three times with PBS containing $0.1 \%$ of Tween 20 and $100 \mu \mathrm{l}$ of a solution containing a peroxidase substrate were added to each well. After 20 minutes, the peroxidase reaction was stopped by adding $50 \mu \mathrm{l}$ of a $2 \mathrm{~N} \mathrm{H}_{2} \mathrm{SO}_{4}$ solution. The colorimetric signal was analyzed (two wavelengths of reading: 450 and $540 \mathrm{~nm}$ ) by using an appropriate spectrophotometry plate reader (Victor V, Perkin Elmer). For calculations, the signal obtained at $540 \mathrm{~nm}$ was subtracted to those obtained at $450 \mathrm{~nm}$.

\subsection{Protein Assay}

Protein assay was performed in cell lysates obtained by sonication, following the method of Bradford [15].

\subsection{Satistics}

Data are expressed as means \pm S.E. of experiments realized at least, in triplicates $(n=3)$. The statistical significances were assessed by Student's t tests or by one way analysis of variance (ANOVA) followed by Holm-Sidak's tests (as indicated).

\section{Results}

We studied the effect of the Uriage thermal water (UTSW) and of an UVB irradiation on the constitutive TauT expression (Figure 1). To determine the effect of UTSW on the baseline expression rate of TauT, human normal keratinocytes were incubated for a 24 and a 72 hours period, in the absence (control) or in the presence of increasing concentrations of UTSW $(0.1 ; 1$ and $10 \%$, $\mathrm{v} / \mathrm{v}$ ). At the indicated time-points (Figure 2), the cell monolayers were rinsed and cell lysates were obtained using ultrasound. TauT was then quantified in these lysates using a home-made ELISA assay (see materials and methods section). As shown in Figure 2, and except for the highest tested concentration after 72 hours of incubation, UTSW did not significantly modify the basal level of TauT expression in normal human keratinocytes.

To address the effect of the UVB on the TauT expression, we irradiated the cells $\left(30 \mathrm{~mJ} / \mathrm{cm}^{2}\right)$ before to follow the above experimental protocol. As shown in figure 3 and as previously described by Rockel et al. in 2007 [16],

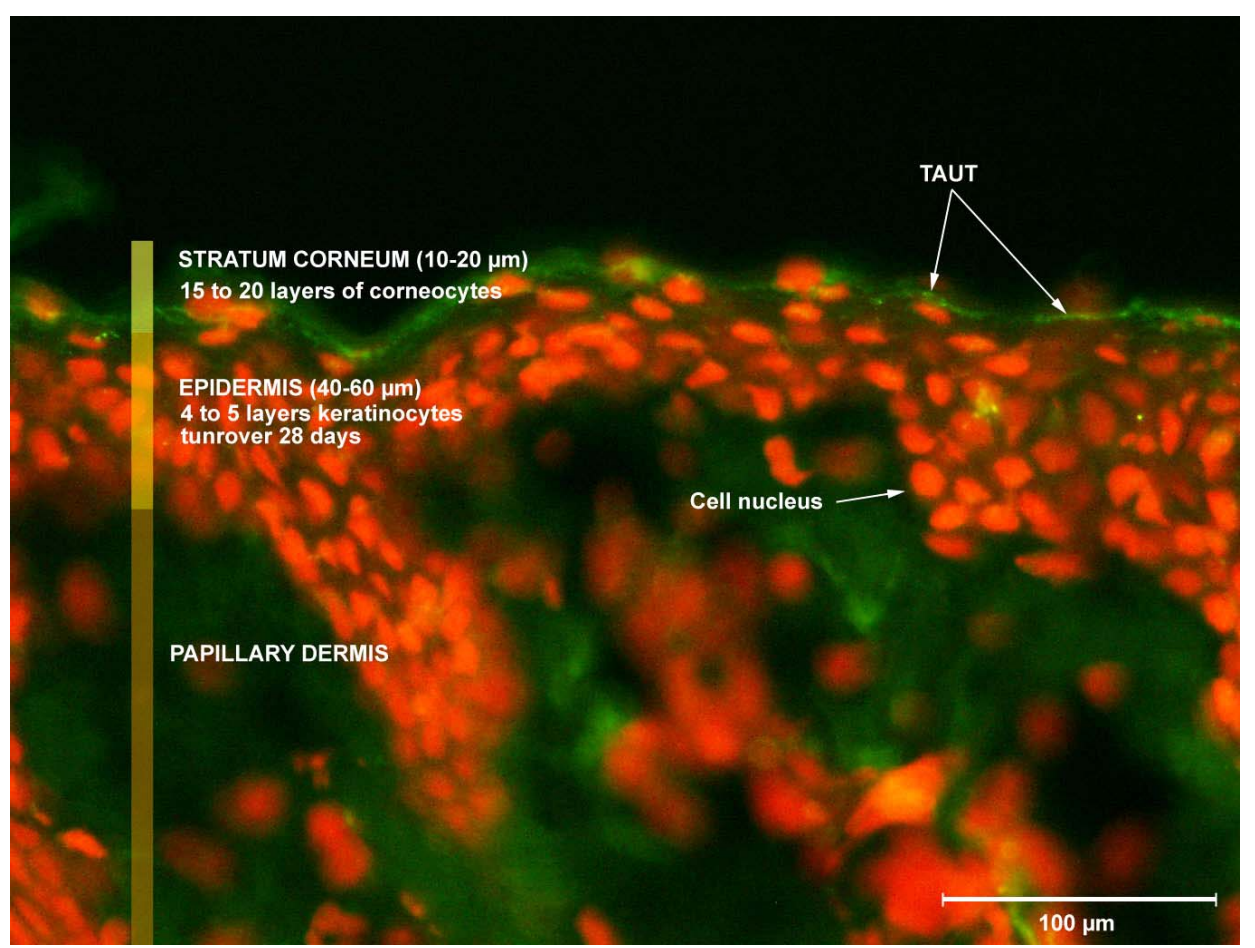

Figure 1. Illustration of TauT expression in human normal skin. 


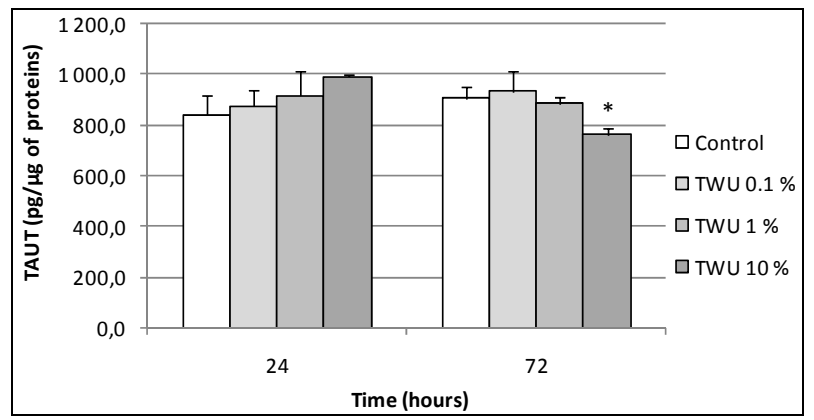

Figure 2. TauT expression in human normal keratinocytes after 24 and 72 hours of treatment with the Uriage thermal spring water. "Significantly different from the "control" condition at the same time-point $(p<0.05$; one way ANOVA + Holm-Sidak test); UTSW, Uriage thermal spring water.

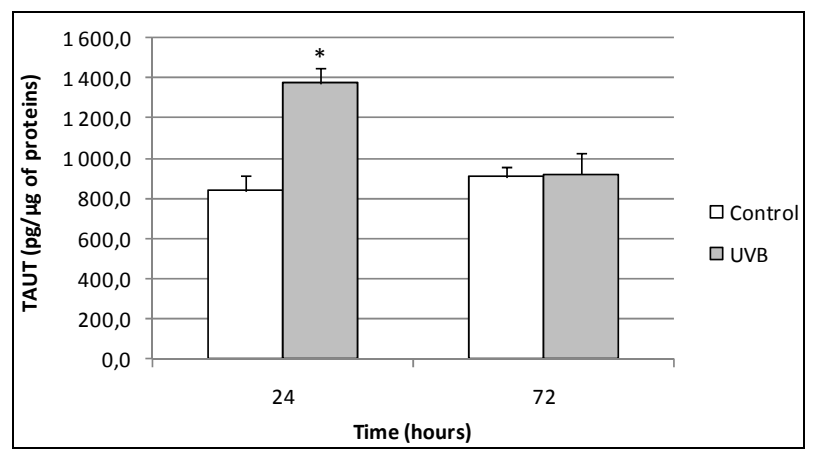

Figure 3. TauT expression in human normal keratinocytes 24 and 72 hours after UVB irradiation. "Significantly different from the "control" condition at the same time-point (p $<0.05$; Student t-test).

when the cells were irradiated by UVB, TauT expression increased after 24 hours, $+63.25 \%(\mathrm{p}<0.05)$ and returned to the basal level after 72 hours. In these conditions, Uriage thermal water slightly reduced the effect of UVB after 24 hours of incubation but above all, permited to maintain a high level of TauT expression after 72 hours of incubation (Figures 4(a) and (b)).

Examining the keratinocyte expression of another Sodium-dependent transporter, we first quantified the SVCT1 constitutive production in cells issued from two young and two aged donors. To perform this quantification, lysates of confluent monolayers of keratinocytes were obtained using ultrasound and SVCT-1 was then assessed in the lysates using a home-made ELISA assay (see materials and methods section). As shown in Figure 5, the basal level of SVCT-1 was significantly higher in young cells: $+43.5 \%(\mathrm{p}<0.01)$.

We next addressed the effect of UVB on the SVCT-1 expression in our models of "young" and "aged" cells. As previously described by Steiling et al. in 2007 [7], UVB significantly decreased the SVCT-1 expression in keratinocytes (Figure 5): $-11.7 \%(p<0.01)$. However, this effect was only observed in the "young" cells and not in the "aged" ones, where a slight increase (non significant) has furthermore been noted (Figure 5).

To address the effect of the UTSW on the SVCT-1 expression in our normal human keratinocytes models, we incubated the cells issued from two "young" and two "aged" donors during 24 hours at $37^{\circ} \mathrm{C}$ in the absence (control) or in the presence of increasing doses $(0.1 ; 1$ and $10 \%, \mathrm{v} / \mathrm{v}$ ) of the thermal water. At the end of the incubation period, the SVCT-1 was quantified in the cell lysates. As shown in Figure 6, UTSW significantly reduced the SVCT-1 expression in "young cells": $-20.9 \%$ $(\mathrm{p}<0.05) ;-26.6 \%(\mathrm{p}<0.05)$ and $-27.0 \%(\mathrm{p}<0.05)$ for UTSW tested at $0.1 ; 1$ and $10 \%(\mathrm{v} / \mathrm{v})$, respectively.

In cells issued from "aged" donors, UTSW presented a biphasic effect. UTSW was so able to significantly increase the SVCT-1 expression when tested at 0.1 and $1 \%$ and to reduce the SVCT-1 expression when tested at 10\% (Figure 6). In the cells issued from the 66 years old donor, the SVCT-1 expression was furthermore increase by UTSW until reach a level superior to those observed in "young" cells (data not shown).

To address the effect of the UTSW on the UVBmodified SVCT-1 expression on our normal human keratinocytes models, we irradiated the cells issued from the two "young" and the two "aged" donors by UVB (30 $\mathrm{mJ} / \mathrm{cm}^{2}$ ) and we next incubated the cells during 24 hours at $37^{\circ} \mathrm{C}$ in the absence (control) or in the presence of increasing doses $(0.1 ; 1$ and $10 \%, \mathrm{v} / \mathrm{v})$ of the thermal water. At the end of the incubation period, the SVCT-1 was quantified in the cell lysates.

As shown in Figure 7, SVCT-1 expression was not longer significantly decreased by UVB in "young" keratinocytes when cells were treated by UTSW at $1 \%$ : $-5.8 \%$ (ns). In the UVB-irradiated "aged" cells, SVCT-1 expression was no more increased by UTSW (Figure 7).

\section{Discussion and Conclusions}

Hydrating cosmetic formulations mainly based their efficacy on oily compounds permitting to avoid transepidermal water loss, but these products also contain water. And even if water has never been shown as a real hydrating compound, we can't exclude that in particular conditions, water could help skin to maintain its hydration rate and furthermore to react in front of various aggressions. In fact, as a lot of membrane transporters, channels, exchangers, have their expression and/or activity partly regulated by several ionic species and as water obviously contains a lot of ions, it is always possible to envisage a role for water in the transport regulation of cellular hydrating elements.

As an example of cellular hydrating elements, there is 


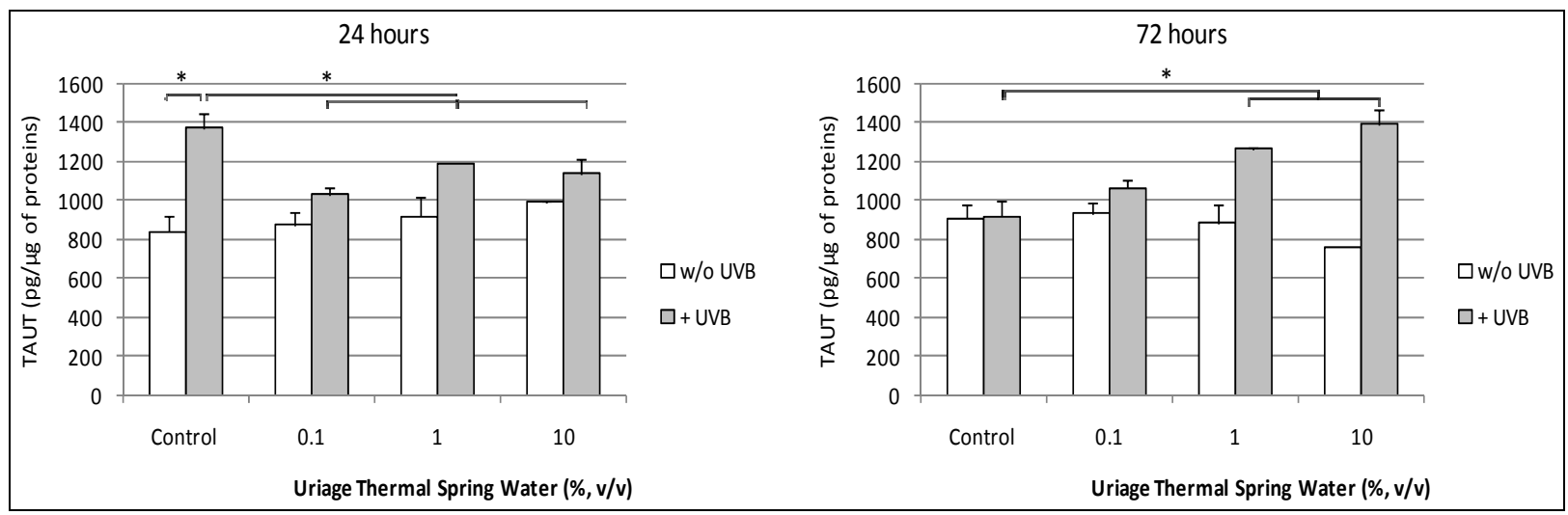

(a)

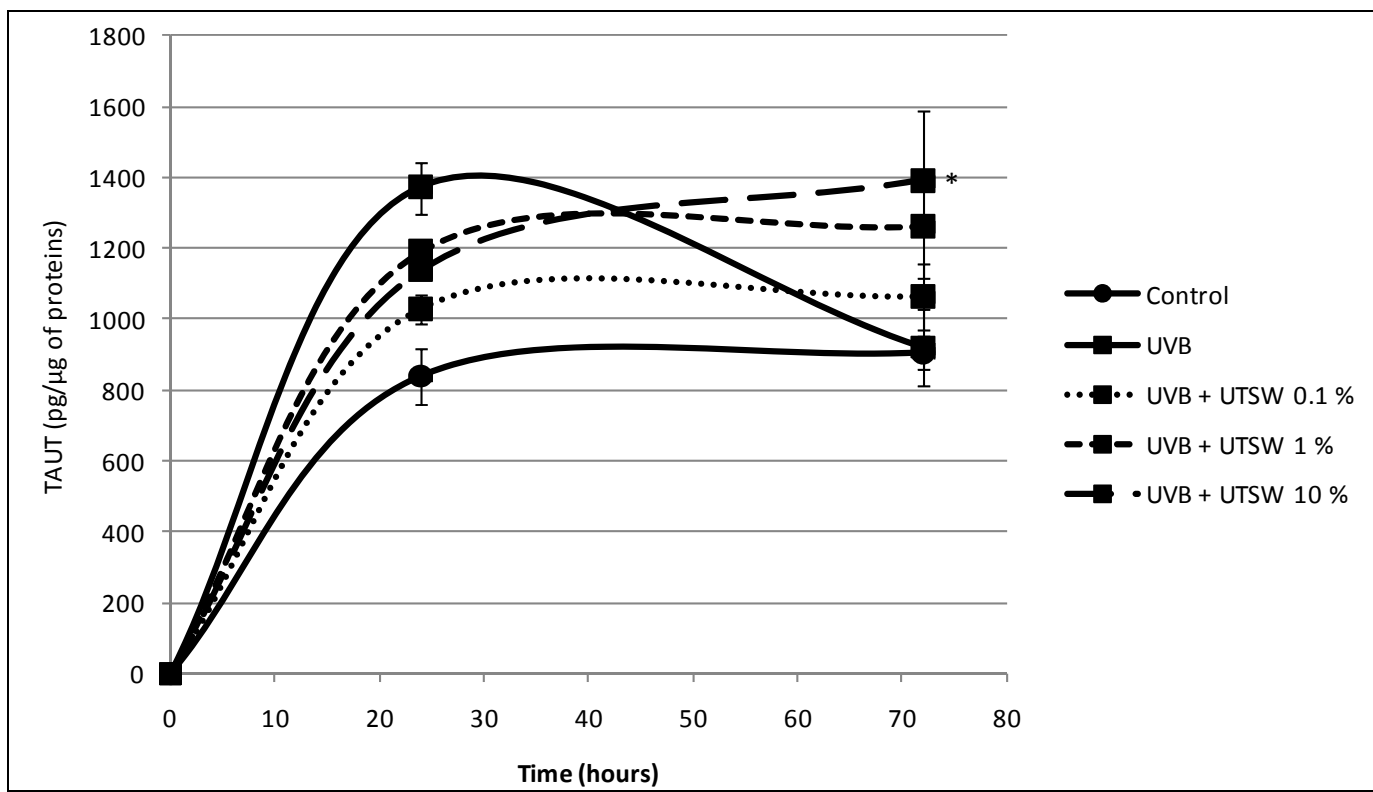

(b)

Figure 4. (a) TauT expression in UVB-irradiated human normal keratinocytes after 24 and 72 hours of treatment with the Uriage thermal spring water. "Significant difference between the conditions indicated by the bars $(p<0.05$; one way ANOVA + Holm-Sidak test); (b) TauT expression in UVB-irradiated human normal keratinocytes after 72 hours of treatment with the Uriage thermal spring water. "Significantly different from the "UVB" condition, at the same end-point $(p<0.05$; one way ANOVA + Holm-Sidak test).

taurine, which is routed into the cell through Sodium-dependent transporters (Taurine Transporters: TauT. TauT expression in skin slide is illustrated in Figure 1) to help skin to fight against hyperosmotic exposure and so dehydration, notably. Thus, Warskulat et al. [17] have demonstrated in 2007 that hyperosmotic exposure of Ha$\mathrm{CaT}$ cells led to an increase in the TauT mRNA expression correlating with an increased taurine uptake, when they compare to normoosmotic controls. In the same manner these authors [17], but also Rockel et al. [16] in a murine model, have shown that UVB are able to increase the TauT expression in keratinocytes. Fitting well with these findings, we also demonstrated that UVB are able to increase TauT expression in our model (Figure 3).
This work so consists in the first demonstration that TauT can be quantified in keratinocytes of normal and human origins and also confirms that UVB are able to increase TauT expression in this model (Figure 3).

Concerning the eventual implication of water in skin hydration, we reasoned that a Sodium-rich water could possibly act through the activation of taurine transporter and/or the regulation of TauT expression. Thus, we incubated human normal keratinocytes with increasing concentrations of Uriage thermal water (UTSW) during 24 and 72 hours before to quantify TauT levels. In these conditions, we noted that UTSW at $10 \%(\mathrm{v} / \mathrm{v})$ induced a slight decrease in TauT expression after 72 hours (Figure 2). 
As Sodium is known to increase taurine influx and as taurine has been reported to down-regulate TauT expression through a negative feedback process [18], we can reasonably explain our observation by these mechanisms.

However, if this result shows that water is not able to increase basal TauT expression, we cannot exclude that Sodium and/or a Sodium-rich water, are able to help skin to fight dehydration by providing the driving force responsible for increased taurine influx. Moreover, it is also possible that in particular conditions, a Sodium-rich water could act on intracellular signaling pathways implicated in the TauT expression regulation.

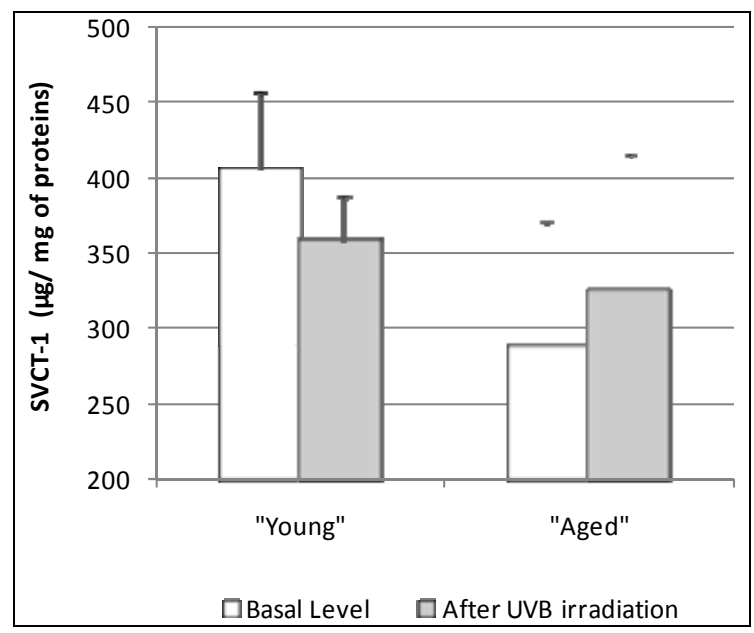

Figure 5. SVCT-1 expression in UVB-irradiated human normal keratinocytes issued from two young and two aged donors. "* Significant difference between the conditions indicated by the bars $\left(p<0.01\right.$; Student t-test). ${ }^{* * *}$ Significant difference between the conditions indicated by the bars $(p<$ 0.001; Student t-test).
Thus, in order to evaluate the effect of UTSW in situation where skin is stressed, we next incubated keratinocytes with UTSW after to have irradiated the cells with UVB.

When cells are "only" irradiated by UVB, a stimulatory effect on TauT expression was observed after 24 hours of incubation but this effect did not endures after 72 hours (Figure 3). In the same way than previously, it is possible to explain this effect by the negative feedback that taurine exerts on TauT expression.

When cells were incubated in the presence of UTSW after the UVB irradiation, we can note that the stimulatory effect of UVB on TauT expression observed at 24 hours, endure after 72 hours of incubation (Figures 4(a) and (b)). Several hypotheses can be proposed to explain this effect.

A first one implies the protein kinase $\mathrm{C}$ (PKC) which controls the TauT activity [19]. Because the PKC is partly regulated by calcium, and because intracellular free calcium quantities vary with the Sodium ones (following passive diffusion mechanisms and through the activity of membrane $\mathrm{Na} / \mathrm{Ca}$ exchangers), it is possible to assume that the PKC activity is lowered by the Sodium entry into the cell. In these conditions, the taurine influx will be also lowered and the negative feedback that taurine exert on TauT expression could be reduced.

A second hypothesis could imply other ions than Sodium. Indeed, the UTSW was not only rich in Sodium but also in zinc for example, a ionic specie implicated in the regulation of the Sodium-dependent transporter SVCT-2 expression [20]. Then, it is possible that zinc can modulate the expression of TauT which is also a Sodium-dependant transporter.

Several others hypothesis could be proposed here and

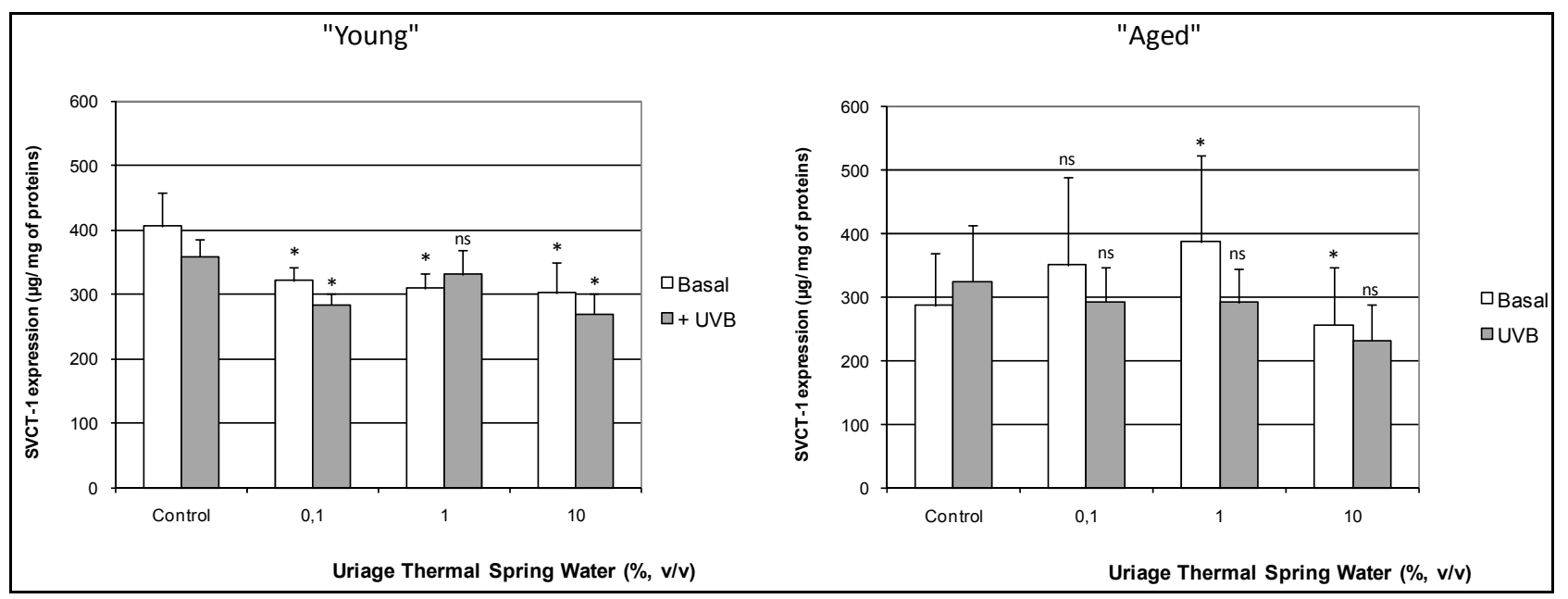

Figure 6. SVCT-1 expression in human normal keratinocytes issued from two young and two aged donors and treated with Uriage thermal spring water. "Significantly different from the "Control" condition ( $p<0.05$; one way ANOVA + Holm-Sidak test). ${ }^{\text {ns }}$ : Non-significantly different from the "Control" condition. 

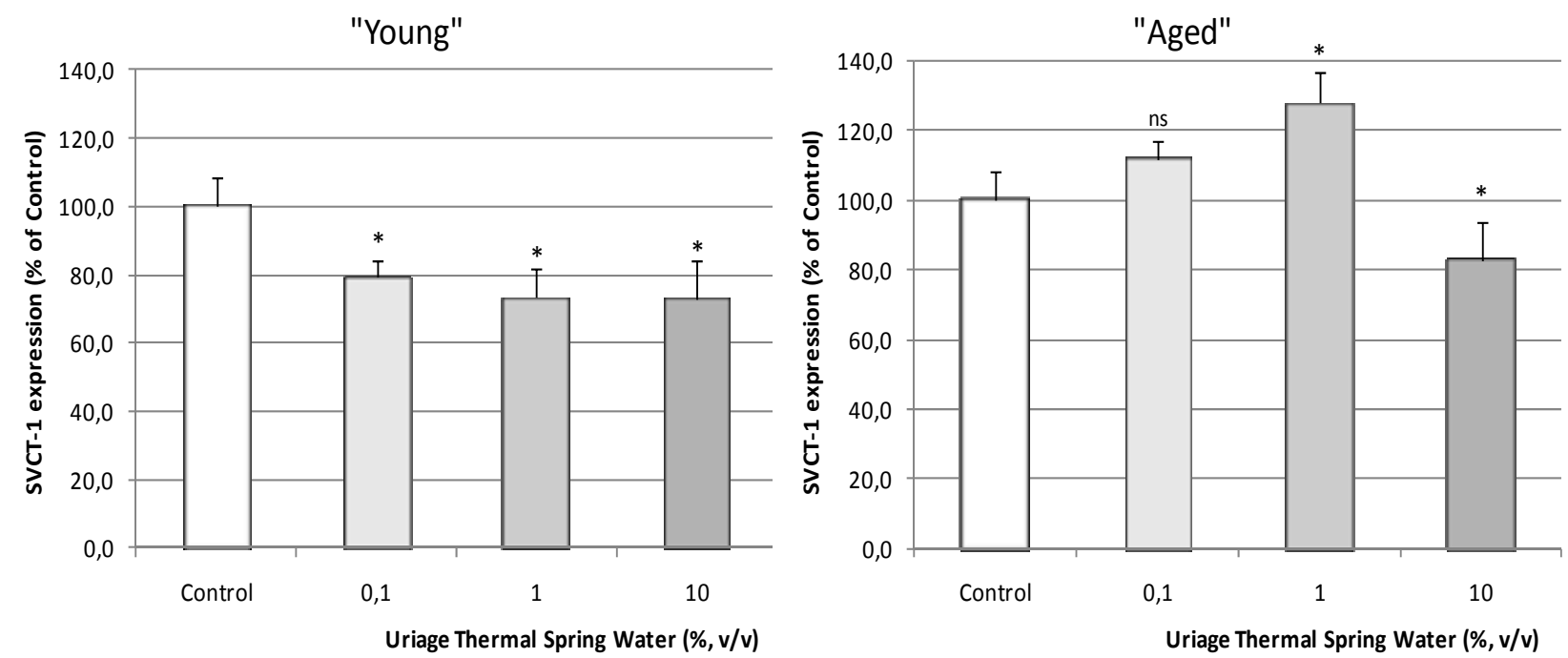

Figure 7. SVCT-1 expression in UVB-irradiated human normal keratinocytes issued from two young and two aged donors, after a 24 hours treatment with the Uriage thermal spring water. ": Significantly different from the corresponding "Control" condition ( $p<0.05$; one way ANOVA + Holm-Sidak test) ${ }^{\text {ns }}$ : Non-significantly different from the corresponding "Control" condition.

further investigations are needed to clarify this issue but we already provide arguments showing that UTSW could possibly help skin to fight against dehydration through an increased taurine influx.

As 1) skin has to fight not only against dehydration but also against other aggressions; and 2) UTSW seems to be able to modulate the expression rate of Sodium-dependent transporters, we examined if UTSW was also able to help skin in front of oxidative stress by acting on the mechanisms regulating the expression of the Sodiumdependent vitamin $\mathrm{C}$ transporter (SVCT).

Two SVCT isoforms are expressed in the skin, SVCT1 and SVCT-2. The SVCT-1 is expressed only in epidermis whereas SVCT-2 is expressed in both epidermis and dermis [7]. Our study concerning human normal keratinocytes, we have then chosen to focus our work on the SVCT-1 isoform (SVCT-1 expression in skin slide is illustrated in Figure 8). Using the ELISA assay that we have especially develop, we then in a first approach, quantified the SVCT-1 in keratinocytes issued from two young and two aged donors.

In our experimental conditions, we observed that the SVCT-1 expression was significantly lower in cells issued from aged donors, compared to the cells issued from the young ones (Figure 5). This result so consists in the first example of an aging dependent SVCT-1 expression in human normal keratinocytes and can give arguments to explain the inverse correlation observed in skin between ascorbic acid levels and increasing age [21].

As UVB irradiations are known to decrease ascorbic acid skin content by acting notably through the repres- sion of the SVCT-1 expression [7], we next addressed this issue in our "young" and "aged" keratinocytes.

Fitting well with the work of Steiling et al. [7], we showed that SVCT-1 was significantly reduced in "young" cells when they were irradiated by UVB (Figure 4).

On the opposite, no inhibitory effect of UVB on SVCT-1 expression was observed in "aged" cells (Figure 5). This last result seems to indicate that skin hold some "shield" mechanisms avoiding the decrease in SVCT-1 expression beyond a certain extent and so guaranteeing a minimal ascorbic acid supplying to fight against particularly stressful situations.

When "young" and "aged" cells were incubated with increasing concentrations of UTSW, opposite results were one more time obtained. Indeed in "young" keratinocytes, a decrease in SVCT-1 expression was observed (Figure 6) whereas a significant increase was noted in "aged" cells treated with UTSW at 1\% (v/v).

In "young" cells however, the effect of UTSW was not dose-related, showing its aspecificity. As we have noted that the total protein levels of our cultures were increased in the presence of UTSW (data not shown), we can reasonably conclude to an effect of UTSW on the cellular proliferation rate. Beyond the study of the UTSW effect on SVCT-1 expression, we so show that UTSW could be trophic enough to ensure a good survival rate in cell culture and suggest that UTSW could also be beneficial for the skin renewal in vivo.

In "aged" cells, we can observe that UTSW at $1 \%$ (v/v) significantly increase the SVCT-1 expression: $+27.8 \%$ (Figure 6). In a very interesting way, this result 


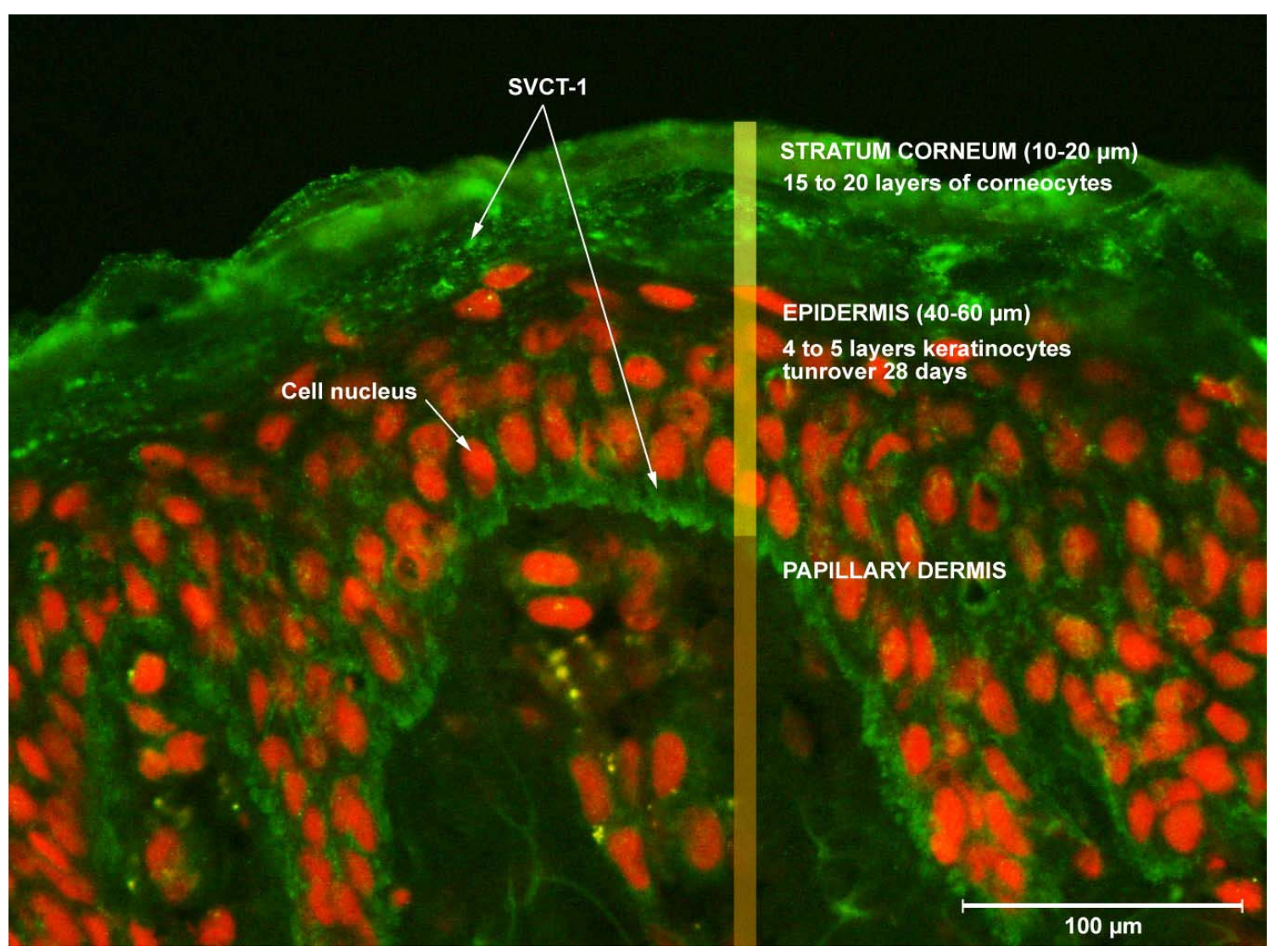

Figure 8. Illustration of SVCT-1 expression in human normal skin.

unambiguously shows that the decreased SVCT-1 expression observed in "aged" cell is, at least, partially reversible. In fact, for one of the two studied donors, the SVCT-1 expression in UTSW treated cells show a higher level to the one observed in "young" cells (data not shown).

The work of Wu et al. in 2003 [20] provides a good rationale to explain this observation. Indeed, these authors have demonstrated that zinc is able to induce the SVCT-2 expression in osteoblasts. As SVCT-1 and -2 show very similar structures and functional characteristics [22], we suggest that the stimulatory effect of UTSW on the SVCT-1 expression could be due to its zinc content, which is relatively high. Taking together, these data suggest that UTSW could help skin to react in front of stresssful situations such as aging.

Finally, we examined the effect of UTSW in UVB-irradiated "young" and "aged" cells. As shown in Figure 7, we can conclude that 1$)$ UTSW at $1 \%(\mathrm{v} / \mathrm{v})$ can acts on the mechanisms implicated in the UVB-repressed expression of SVCT-1 in "young" cells, providing by this way a protective effect in front of solar irradiations; and 2) As the effect of the thermal water on SVCT-1 expression in "aged" cells did not endure, that UTSW is not strong enough to "resist" in front "aging" plus "UVB ir- radiation".

In conclusion, we demonstrated for the first time that it was possible to quantify two Sodium-dependent transporters (TauT and SVCT-1) using ELISA assays in human normal keratinocytes. We show that in the selected experimental conditions, TauT and SVCT-1 expressions are modulated by UVB. We provide the first example for an altered SVCT-1 expression with aging in human normal epithelial skin cells. And at last but not at least, we provide arguments suggesting that a thermal water (the Uriage thermal water) could possibly help the skin to fight against stressful situations such as dehydration, UVB irradiation and aging.

\section{Acknowledgements}

The authors are very thankful to BIO-EC for very good quality illustrations of TauT and SVCT-1 cutaneous expression. The authors would also like to thank to the Doctor Toni Ionescu for his rapid and understanding linguistic support.

\section{REFERENCES}

[1] H. Morrison, "Photochemistry and Photobiology of Uro- 
canic Acid," Photochemistry and Photobiology, Vol. 43, No. 6, 1986, pp. 663-665.

[2] Y. Miyamura, et al., "Regulation of Human Skin Pigmentation and Responses to Ultraviolet Radiation," Pigment Cell Research, Vol. 20, No. 1, 2007, pp. 2-13. doi:10.1111/j.1600-0749.2006.00358.x

[3] B. E. Johnson, "The Influence of Radiation on the Skin and the Basis of Protection," International Journal of Cosmetic Science, Vol. 5, No. 4, 1983, pp. 131-139. doi:10.1111/j.1467-2494.1983.tb00334.x

[4] R. Kohen, "Skin Antioxidants: Their Role in Aging and in Oxidative Stress - New Approaches for Their Evaluation," Biomedicine \& Pharmacotherapy, Vol. 53, No. 4, 1999, pp. 181-192. doi:10.1016/S0753-3322(99)80087-0

[5] C. Cao, et al., "All-Trans Retinoic Acid Attenuates Ultraviolet Radiation-Induced Down-Regulation of Aquaporin-3 and Water Permeability in Human Keratinocytes," Journal of Cellular Physiology, Vol. 215, No. 2, 2008, pp. 506-516. doi:10.1002/jep.21336

[6] G. Janeke, et al., "Role of Taurine Accumulation in Keratinocyte Hydration," Journal of Investigative Dermatology, Vol. 12, No. 2, 2003, pp. 354-361. doi:10.1046/j.1523-1747.2003.12366.x

[7] H. Steiling, et al., "Sodium-Dependent Vitamin C Transporter Isoforms in Skin: Distribution, Kinetics, and Effect of UVB-Induced Oxidative Stress," Free Radical Biology and Medicine, Vol. 43, No. 5, 2007, pp. 752-762. doi:10.1016/j.freeradbiomed.2007.05.001

[8] F. Grafe, W. Wohlrab, R. H. Neubert and M. Brandsch, "Functional Characterization of Sodium- and ChlorideDependent Taurine Transport in Human Keratinocytes," European Journal of Pharmaceutics and Biopharmaceutics, Vol. 57, No. 2, 2004, pp. 337-341. doi:10.1016/j.ejpb.2003.10.010

[9] X. Wu, et al., "Zinc-Induced Sodium-Dependent Vitamin C Transporter 2 Expression: Potent Roles in Osteoblast Differentiation," Archives of Biochemistry and Biophysics, Vol. 420, No. 1, 2003, pp. 114-120. doi:10.1016/j.abb.2003.09.013

[10] H. Qiao and J. M. May, "Development of Ascorbate Transporters in Brain Cortical Capillary Endothelial Cells in Culture," Brain Research, Vol. 1208, 2008, pp. 79-86. doi:10.1016/j.brainres.2008.02.102

[11] K. Erikson and M. Aschner, "Manganese Causes Differential Regulation of Glutamate Transporter (GLAST) Taurine Transporter and Metallothionein in Cultured Rat Astrocytes," Neurotoxicology, Vol. 23, No. 4-5, 2003, pp. 595-602. doi:10.1016/S0161-813X(02)00012-8

[12] G. H. Jin, Y. Liu, S. Z. Jin, X. D. Liu and S. Z. Liu, "UVB Induced Oxidative Stress in Human Keratinocytes and Protective Effect of Antioxidant Agents," Radiation and Environmental Biophysics, Vol. 46, No. 1, 2007, pp. 61-68. doi:10.1007/s00411-007-0096-1

[13] Y. Uchida, M. Behne, D. Quiec, P. M. Elias and W. M. Holleran, "Vitamin C Stimulates Sphingolipid Production and Markers of Barrier Formation in Submerged Human Keratinocyte Cultures," Journal of Investigative Dermatology, Vol. 117, No. 5, 2001, pp. 1307-1313. doi:10.1046/i.0022-202x.2001.01555.x

[14] W. E. Parish, J. Read and S. E. Paterson, "Changes in Basal Cell Mitosis and Transepidermal Water Loss in Skin Cultures Treated with Vitamins C and E," Experimental Dermatology, Vol. 14, No. 9, 2005, pp. 684-691. doi:10.1111/j.0906-6705.2005.00340.x

[15] M. Bradford, "A Rapid and Sensitive Method for the Quantitation of Microgram Quantities of Protein Utilizing the Principle of Protein-Dye Binding," Analytical Biochemistry, Vol. 72, No. 1-2, 1976, pp. 248-254. doi:10.1016/0003-2697(76)90527-3

[16] N. Rockel, et al., "The Osmolyte Taurine Protects against Ultraviolet B Radiation-Induced Immunosuppression," Journal of Immunology, Vol. 179, No. 6, 2007, pp. 36043612 .

[17] U. Warskulat, S. Brookmann, A. Reinen and D. Häussinger, "Ultraviolet B Radiation Induces Cell Shrinkage and Increases Osmolyte Transporter mRNA Expression and Osmolyte Uptake in HaCaT Keratinocytes," Biological Chemistry, Vol. 388, No. 12, 2007, pp. 1345-1352. doi:10.1515/BC.2007.140

[18] M. L. Tappaz, "Taurine Biosynthetic Enzymes and Taurine Transporter: Molecular Identification and Regulations," Neurochemical Research, Vol. 29, No. 1, 2004, pp. 83-96. doi:10.1023/B:NERE.0000010436.44223.f8

[19] X. Han, A. B. Patters, D. P. Jones, I. Zelikovic and R. W. Chesney, "The Taurine Transporter: Mechanisms of Regulation," Acta Physiologica, Vol. 187, No. 1-2, 2006, pp. 61-73. doi:10.1111/j.1748-1716.2006.01573.x

[20] X. Wu, et al., "Zinc-Induced Sodium-Dependent Vitamin C Transporter 2 Expression: Potent Roles in Osteoblast Differentiation," Archives of Biochemistry and Biophysics, Vol. 420, No. 1, 2003, pp. 114-120. doi:10.1016/j.abb.2003.09.013

[21] N. Leveque, S. Robin, S. Makki, P. Muret, A. Rougier and P. Humbert, "Iron and Ascorbic Acid Concentrations in Human Dermis with Regard to Age and Body Sites," Gerontology, Vol. 49, No. 2, 2003, pp. 117-122. doi: $10.1159 / 000067951$

[22] I. Savini, A. Rossi, C. Pierro, L. Avigliano and M. V. Catani, "SVCT1 and SVCT2: Key Proteins for Vitamin C Uptake," Amino Acids, Vol. 34, No. 3, 2008, pp. 347-355. doi:10.1007/s00726-007-0555-7 